\title{
The group of automorphisms of the Lie algebra of derivations of a field of rational functions
}

\author{
V. V. Bavula
}

\begin{abstract}
We prove that the group of automorphisms of the Lie algebra $\operatorname{Der}_{K}\left(Q_{n}\right)$ of derivations of the field of rational functions $Q_{n}=K\left(x_{1}, \ldots, x_{n}\right)$ over a field of characteristic zero is canonically isomorphic to the group of automorphisms of the $K$-algebra $Q_{n}$.

Key Words: Group of automorphisms, monomorphism, Lie algebra, automorphism, locally nilpotent derivation, the field of rational functions in $n$ variables.

Mathematics subject classification 2010: 17B40, 17B20, 17B66, 17B65, 17 B30.
\end{abstract}

\section{Introduction}

In this paper, module means a left module, $K$ is a field of characteristic zero and $K^{*}$ is its group of units, and the following notation is fixed:

- $P_{n}:=K\left[x_{1}, \ldots, x_{n}\right]=\bigoplus_{\alpha \in \mathbb{N}^{n}} K x^{\alpha}$ is a polynomial algebra over $K$ where $x^{\alpha}:=x_{1}^{\alpha_{1}} \cdots x_{n}^{\alpha_{n}}$ and $Q_{n}:=K\left(x_{1}, \ldots, x_{n}\right)$ is the field of rational functions,

- $G_{n}:=\operatorname{Aut}_{\mathrm{K}-\mathrm{alg}}\left(P_{n}\right)$ and $\mathbb{Q}_{n}:=\operatorname{Aut}_{\mathrm{K}-\mathrm{alg}}\left(Q_{n}\right)$;

- $\partial_{1}:=\frac{\partial}{\partial x_{1}}, \ldots, \partial_{n}:=\frac{\partial}{\partial x_{n}}$ are the partial derivatives (K-linear derivations) of $P_{n}$,

- $D_{n}:=\operatorname{Der}_{K}\left(P_{n}\right)=\bigoplus_{i=1}^{n} P_{n} \partial_{i} \subseteq E_{n}:=\operatorname{Der}_{K}\left(Q_{n}\right)=\bigoplus_{i=1}^{n} Q_{n} \partial_{i}$ are the Lie algebras of $K$-derivations of $P_{n}$ and $Q_{n}$ respectively where $[\partial, \delta]:=\partial \delta-\delta \partial$,

- $\mathbb{G}_{n}:=\operatorname{Aut}_{\text {Lie }}\left(D_{n}\right)$ and $\mathbb{E}_{n}:=\operatorname{Aut}_{\text {Lie }}\left(E_{n}\right)$,

- $\delta_{1}:=\operatorname{ad}\left(\partial_{1}\right), \ldots, \delta_{n}:=\operatorname{ad}\left(\partial_{n}\right)$ are the inner derivations of the Lie algebras $D_{n}$ and $E_{n}$ where $\operatorname{ad}(a)(b):=[a, b]$,

- $\mathcal{D}_{n}:=\bigoplus_{i=1}^{n} K \partial_{i}$,

- $\mathcal{H}_{n}:=\bigoplus_{i=1}^{n} K H_{i}$ where $H_{1}:=x_{1} \partial_{1}, \ldots, H_{n}:=x_{n} \partial_{n}$,

- for each natural number $n \geq 2, \mathfrak{u}_{n}:=K \partial_{1}+P_{1} \partial_{2}+\cdots+P_{n-1} \partial_{n}$ is the Lie algebra of triangular polynomial derivations (it is a Lie subalgebra of $D_{n}$ ) and $\operatorname{Aut}_{\text {Lie }}\left(\mathfrak{u}_{n}\right)$ is its group of automorphisms.

Theorem 1.1 [4] $\mathbb{G}_{n}=G_{n}$.

The aim of the paper is to prove the following theorem.

Theorem 1.2 $\mathbb{E}_{n}=\mathbb{Q}_{n}$. 
Structure of the proof. (i) $\mathbb{Q}_{n} \subseteq \mathbb{E}_{n}$ via the group monomorphism (Lemma 2.3 and (3))

$$
\mathbb{Q}_{n} \rightarrow \mathbb{E}_{n}, \quad \sigma \mapsto \sigma: \partial \mapsto \sigma(\partial):=\sigma \partial \sigma^{-1} .
$$

(ii) Let $\sigma \in \mathbb{E}_{n}$. Then $\partial_{1}^{\prime}:=\sigma\left(\partial_{1}\right), \ldots, \partial_{n}^{\prime}:=\sigma\left(\partial_{n}\right)$ are commuting derivations of $Q_{n}$ such that $E_{n}=\bigoplus_{i=1}^{n} Q_{n} \partial_{i}^{\prime}$ (Lemma 2.12, (2)) and

(iii) $\bigcap_{i=1}^{n} \operatorname{ker}_{Q_{n}}\left(\partial_{i}^{\prime}\right)=K$ (Lemma 2.12.(1)).

(iv)(crux) There exist elements $x_{1}^{\prime}, \ldots, x_{n}^{\prime} \in Q_{n}$ such that $\partial_{i}^{\prime}\left(x_{j}^{\prime}\right)=\delta_{i j}$ for $i, j=1, \ldots, n$ (Lemma 2.12,(3)).

(v) $\sigma\left(x^{\alpha} \partial_{i}\right)=x^{\prime \alpha} \partial_{i}^{\prime}$ for all $\alpha \in \mathbb{N}^{n}$ and $i=1, \ldots, n$ (Lemma 2.12,(6)).

(vi) The $K$-algebra homomorphism $\sigma^{\prime}: Q_{n} \rightarrow Q_{n}, x_{i} \mapsto x_{i}^{\prime}, i=1, \ldots, n$ is an automorphism such that $\sigma^{\prime}\left(q \partial_{i}\right)=\sigma^{\prime}(q) \partial_{i}^{\prime}$ for all $q \in Q_{n}$ and $i=1, \ldots, n$.

(vii) $\operatorname{Fix}_{\mathbb{E}_{n}}\left(\partial_{1}, \ldots, \partial_{n}, H_{1}, \ldots, H_{n}\right)=\{e\}$ (Proposition 2.9.(1)). Hence, $\sigma=\sigma^{\prime} \in \mathbb{Q}_{n}$, by (v) and (vi), i.e. $\mathbb{E}_{n}=\mathbb{Q}_{n}$.

The groups of automorphisms of the Lie algebras $D_{n}$ and $\mathfrak{u}_{n}$.

Theorem 1.3 (Theorem 5.3, 3] Aut $_{\text {Lie }}\left(\mathfrak{u}_{n}\right) \simeq \mathbb{T}^{n} \ltimes\left(\operatorname{UAut}_{K}\left(P_{n}\right)_{n} \rtimes\left(\mathbb{F}_{n}^{\prime} \times \mathbb{E}_{n}\right)\right)$ where $\mathbb{T}^{n}$ is an algebraic n-dimensional torus, $\operatorname{UAut}_{K}\left(P_{n}\right)_{n}$ is an explicit factor group of the group $\operatorname{UAut}_{K}\left(P_{n}\right)$ of unitriangular polynomial automorphisms, $\mathbb{F}_{n}^{\prime}$ and $\mathbb{E}_{n}$ are explicit groups that are isomorphic respectively to the groups $\mathbb{I}$ and $\mathbb{J}^{n-2}$ where $\mathbb{I}:=\left(1+t^{2} K[[t]], \cdot\right) \simeq K^{\mathbb{N}}$ and $\mathbb{J}:=(t K[[t]],+) \simeq K^{\mathbb{N}}$.

Comparing the groups $\mathbb{G}_{n}, \mathbb{E}_{n}$ and $\operatorname{Aut}_{\text {Lie }}\left(\mathfrak{u}_{n}\right)$ we see that the group $\operatorname{UAut}_{K}\left(P_{n}\right)_{n}$ of polynomial automorphisms is a tiny part of the group $\operatorname{Aut}_{\text {Lie }}\left(\mathfrak{u}_{n}\right)$ but in contrast $\mathbb{G}_{n}=G_{n}$ and $\mathbb{E}_{n}=\mathbb{Q}_{n}$.

Theorem 1.4 [1] Every monomorphism of the Lie algebra $\mathfrak{u}_{n}$ is an automorphism.

Not every epimorphism of the Lie algebra $\mathfrak{u}_{n}$ is an automorphism. Moreover, there are countably many distinct ideals $\left\{I_{i \omega^{n-1}} \mid i \geq 0\right\}$ such that

$$
I_{0}=\{0\} \subset I_{\omega^{n-1}} \subset I_{2 \omega^{n-1}} \subset \cdots \subset I_{i \omega^{n-1}} \subset \cdots
$$

and the Lie algebras $\mathfrak{u}_{n} / I_{i \omega^{n-1}}$ and $\mathfrak{u}_{n}$ are isomorphic (Theorem 5.1.(1), [2]).

Conjecture, 4]. Every homomorphism of the Lie algebra $D_{n}$ is an automorphism.

The groups of automorphisms of the Witt $W_{n}(n \geq 2)$ and the Virasoro Vir Lie algebras were found in 5 .

\section{Proof of Theorem 1.2}

This section can be seen as a proof of Theorem 1.2. The proof is split into several statements that reflect 'Structure of the proof of Theorem [1.2] given in the Introduction.

Let $\mathcal{G}$ be a Lie algebra and $\mathcal{H}$ be its Lie subalgebra. The centralizer $C_{\mathcal{G}}(\mathcal{H}):=\{x \in \mathcal{G} \mid[x, \mathcal{H}]=$ $0\}$ of $\mathcal{H}$ in $\mathcal{G}$ is a Lie subalgebra of $\mathcal{G}$. In particular, $Z(\mathcal{G}):=C_{\mathcal{G}}(\mathcal{G})$ is the centre of the Lie algebra $\mathcal{G}$. The normalizer $N_{\mathcal{G}}(\mathcal{H}):=\{x \in \mathcal{G} \mid[x, \mathcal{H}] \subseteq \mathcal{H}\}$ of $\mathcal{H}$ in $\mathcal{G}$ is a Lie subalgebra of $\mathcal{G}$, it is the largest Lie subalgebra of $\mathcal{G}$ that contains $\mathcal{H}$ as an ideal.

Let $V$ be a vector space over $K$. A $K$-linear map $\delta: V \rightarrow V$ is called a locally nilpotent map if $V=\bigcup_{i \geq 1} \operatorname{ker}\left(\delta^{i}\right)$ or, equivalently, for every $v \in V, \delta^{i}(v)=0$ for all $i \gg 1$. When $\delta$ is a locally nilpotent map in $V$ we also say that $\delta$ acts locally nilpotently on $V$. Every nilpotent linear map 
$\delta$, that is $\delta^{n}=0$ for some $n \geq 1$, is a locally nilpotent map but not vice versa, in general. Let $\mathcal{G}$ be a Lie algebra. Each element $a \in \mathcal{G}$ determines the derivation of the Lie algebra $\mathcal{G}$ by the rule $\operatorname{ad}(a): \mathcal{G} \rightarrow \mathcal{G}, b \mapsto[a, b]$, which is called the inner derivation associated with $a$. The $\operatorname{set} \operatorname{Inn}(\mathcal{G})$ of all the inner derivations of the Lie algebra $\mathcal{G}$ is a Lie subalgebra of the Lie algebra $\left(\operatorname{End}_{K}(\mathcal{G}),[\cdot, \cdot]\right)$ where $[f, g]:=f g-g f$. There is the short exact sequence of Lie algebras

$$
0 \rightarrow Z(\mathcal{G}) \rightarrow \mathcal{G} \stackrel{\text { ad }}{\rightarrow} \operatorname{Inn}(\mathcal{G}) \rightarrow 0
$$

that is $\operatorname{Inn}(\mathcal{G}) \simeq \mathcal{G} / Z(\mathcal{G})$ where $Z(\mathcal{G})$ is the centre of the Lie algebra $\mathcal{G}$ and $\operatorname{ad}([a, b])=[\operatorname{ad}(a), \operatorname{ad}(b)]$ for all elements $a, b \in \mathcal{G}$. An element $a \in \mathcal{G}$ is called a locally nilpotent element (respectively, a nilpotent element) if so is the inner derivation $\operatorname{ad}(a)$ of the Lie algebra $\mathcal{G}$.

The Lie algebra $E_{n}$. Since

$$
E_{n}=\bigoplus_{i=1}^{n} Q_{n} \partial_{i}=\bigoplus_{i=1}^{n} Q_{n} H_{i}
$$

every element $\partial \in E_{n}$ is a unique sum $\partial=\sum_{i=1}^{n} a_{i} \partial_{i}=\sum_{i=1}^{n} b_{i} H_{i}$ where $a_{i}=x_{i} b_{i} \in Q_{n}$. The field $Q_{n}$ is the union $\bigcup_{0 \neq f \in P_{n}} P_{n, f}$ where $P_{n, f}$ is the localization of $P_{n}$ at the powers of $f$. The algebra $Q_{n}$ is a localization of $P_{n, f}$. Hence $D_{n, f}:=\operatorname{Der}_{K}\left(P_{n, f}\right)=\bigoplus_{i=1}^{n} P_{n, f} \partial_{i} \subseteq E_{n}$ and

$$
E_{n}=\bigcup_{0 \neq f \in P_{n}} D_{n, f}
$$

$Q_{n}$ is an $E_{n}$-module. The field $Q_{n}$ is a (left) $E_{n}$-module: $E_{n} \times Q_{n} \rightarrow Q_{n},(\partial, q) \mapsto \partial * q$. In more detail, if $\partial=\sum_{i=1}^{n} a_{i} \partial_{i}$ where $a_{i} \in Q_{n}$ then

$$
\partial * q=\sum_{i=1}^{n} a_{i} \frac{\partial q}{\partial x_{i}} .
$$

The $E_{n}$-module $Q_{n}$ is not a simple module since $K$ is an $E_{n}$-submodule of $Q_{n}$, and

$$
\bigcap_{i=1}^{n} \operatorname{ker}_{Q_{n}}\left(\partial_{i}\right)=K
$$

Lemma 2.1 The $E_{n}$-module $Q_{n} / K$ is simple with $\operatorname{End}_{E_{n}}\left(Q_{n} / K\right)=K$ id where id is the identity map.

Proof. We have to show that for each non-scalar rational function, say $p q^{-1} \in Q_{n}$, the $E_{n^{-}}$ submodule $M$ of $Q_{n} / K$ it generates coincides with the $E_{n}$-module $Q_{n} / K$. By (2), $a_{i}=\partial_{i} *$ $\left(p q^{-1}\right) \neq 0$ for some $i$. Then for all elements $u \in Q_{n}, u a_{i}^{-1} \partial_{i} *\left(p q^{-1}+K\right)=u+K$. So, $Q_{n} / K$ is a simple $E_{n}$-module. Let $f \in \operatorname{End}_{E_{n}}\left(Q_{n} / K\right)$. Then applying $f$ to the equalities $\partial_{i} *\left(x_{1}+K\right)=\delta_{i 1}$ for $i=1, \ldots, n$, we obtain the equalities

$$
\partial_{i} * f\left(x_{1}+K\right)=\delta_{i 1} \text { for } i=1, \ldots, n .
$$

Hence, $f\left(x_{1}+K\right) \in \bigcap_{i=2}^{n} \operatorname{ker}_{Q_{n} / K}\left(\partial_{i}\right) \cap \operatorname{ker}_{Q_{n} / K}\left(\partial_{i}^{2}\right)=\left(K\left(x_{1}\right) / K\right) \cap \operatorname{ker}_{Q_{n} / K}\left(\partial_{i}^{2}\right)=K\left(x_{1}+K\right)$. So, $f\left(x_{1}+K\right)=\lambda\left(x_{1}+K\right)$ and so $f=\lambda$ id, by the simplicity of the $E_{n}$-module $Q_{n} / K$.

The Cartan subalgebra $\mathcal{H}_{n}$ of $E_{n}$. A nilpotent Lie subalgebra $C$ of a Lie algebra $\mathcal{G}$ is called a Cartan subalgebra of $\mathcal{G}$ if it coincides with its normalizer. We use often the following obvious observation: An abelian Lie subalgebra that coincides with its centralizer is a maximal abelian Lie subalgebra.

Lemma 2.2 1. $\mathcal{H}_{n}$ is a Cartan subalgebra of $E_{n}$. 
2. $\mathcal{H}_{n}=C_{E_{n}}\left(\mathcal{H}_{n}\right)$ is a maximal abelian Lie subalgebra of $E_{n}$.

Proof. 2. Clearly, $\mathcal{H}_{n} \subseteq C_{E_{n}}\left(\mathcal{H}_{n}\right)$. Let $\partial=\sum_{i=1}^{n} a_{i} H_{i} \in C_{E_{n}}\left(\mathcal{H}_{n}\right)$ where $a_{i} \in Q_{n}$. Then all $a_{i} \in \cap_{i=1}^{n} \operatorname{ker}_{Q_{n}}\left(H_{i}\right)=\cap_{i=1}^{n} \operatorname{ker}_{Q_{n}}\left(\partial_{i}\right)=K$, by (2), and so $\partial \in \mathcal{H}_{n}$. Therefore, $\mathcal{H}_{n}=C_{E_{n}}\left(\mathcal{H}_{n}\right)$ is a maximal abelian Lie subalgebra of $E_{n}$.

1. By statement 2, we have to show that $\mathcal{H}_{n}=N:=N_{E_{n}}\left(\mathcal{H}_{n}\right)$. Let $\partial=\sum_{i=1}^{n} a_{i} H_{i} \in N$, we have to show that all $a_{i} \in K$. By statement 2, for all $j=1, \ldots, n, \mathcal{H}_{n} \ni\left[H_{j}, \partial\right]=\sum_{i=1}^{n} H_{j}\left(a_{i}\right) H_{i}$, and so $H_{j}\left(a_{i}\right) \in K$ for all $i$ and $j$. This condition holds if all $a_{i} \in K$, i.e. $\partial \in \mathcal{H}_{n}$. Suppose that $a_{i} \notin K$ for some $i$, we seek a contradiction. Then necessarily, $a_{i} \notin K\left(x_{1}, \ldots, \widehat{x}_{j}, \ldots, x_{n}\right)$ for some $j$. Since $Q_{n}=K\left(x_{1}, \ldots, \widehat{x}_{j}, \ldots, x_{n}\right)\left(x_{j}\right)$, the result follows from the following claim.

Claim: If $a \in K(x) \backslash K$ then $H(a) \notin K$. The field $K(x)$ is a subfield of the series field $K((x)):=\left\{\sum_{i>-\infty} \lambda_{i} x^{i} \mid \lambda_{i} \in K\right\}$. Since $H\left(\sum_{i>-\infty} \lambda_{i} x^{i}\right)=\sum_{i>-\infty} i \lambda_{i} x^{i}$, the Claim is obvious. Then, by the Claim, $H_{j}\left(a_{i}\right) \notin K$, a contradiction.

Lemma 2.3 [5] Let $R$ be a commutative ring such that there exists a derivation $\partial \in \operatorname{Der}(R)$ such that $r \partial \neq 0$ for all nonzero elements $r \in R$ (eg, $R=P_{n}, Q_{n}$ and $\left.\delta=\partial_{1}\right)$. Then the group homomorphism

$$
\operatorname{Aut}(R) \rightarrow \operatorname{Aut}_{\mathrm{Lie}}(\operatorname{Der}(R)), \quad \sigma \mapsto \sigma: \delta \mapsto \sigma(\delta):=\sigma \delta \sigma^{-1},
$$

is a monomorphism.

The $\mathbb{Q}_{n}$-module $E_{n}$. The Lie algebra $E_{n}$ is a $\mathbb{Q}_{n}$-module,

$$
\mathbb{Q}_{n} \times E_{n} \rightarrow E_{n}, \quad(\sigma, \partial) \mapsto \sigma(\partial):=\sigma \partial \sigma^{-1} .
$$

By Lemma 2.3 , the $\mathbb{Q}_{n}$-module $E_{n}$ is faithful and the map

$$
\mathbb{Q}_{n} \rightarrow \mathbb{E}_{n}, \quad \sigma \mapsto \sigma: \partial \mapsto \sigma(\partial)=\sigma \partial \sigma^{-1},
$$

is a group monomorphism. We identify the group $\mathbb{Q}_{n}$ with its image in $\mathbb{E}_{n}, \mathbb{Q}_{n} \subseteq \mathbb{E}_{n}$. Every automorphism $\sigma \in \mathbb{Q}_{n}$ is uniquely determined by the elements

$$
x_{1}^{\prime}:=\sigma\left(x_{1}\right), \ldots, x_{n}^{\prime}:=\sigma\left(x_{n}\right) .
$$

Let $M_{n}\left(Q_{n}\right)$ be the algebra of $n \times n$ matrices over $Q_{n}$. The matrix $J(\sigma):=\left(J(\sigma)_{i j}\right) \in M_{n}\left(Q_{n}\right)$, where $J(\sigma)_{i j}=\frac{\partial x_{j}^{\prime}}{\partial x_{i}}$, is called the Jacobian matrix of the automorphism (endomorphism) $\sigma$ and its determinant $\mathcal{J}(\sigma):=\operatorname{det} J(\sigma)$ is called the Jacobian of $\sigma$. So, the $j$ 'th column of $J(\sigma)$ is the gradient $\operatorname{grad} x_{j}^{\prime}:=\left(\frac{\partial x_{j}^{\prime}}{\partial x_{1}}, \ldots, \frac{\partial x_{j}^{\prime}}{\partial x_{n}}\right)^{T}$ of the polynomial $x_{j}^{\prime}$. Then the derivations

$$
\partial_{1}^{\prime}:=\sigma \partial_{1} \sigma^{-1}, \ldots, \partial_{n}^{\prime}:=\sigma \partial_{n} \sigma^{-1}
$$

are the partial derivatives of $Q_{n}$ with respect to the variables $x_{1}^{\prime}, \ldots, x_{n}^{\prime}$,

$$
\partial_{1}^{\prime}=\frac{\partial}{\partial x_{1}^{\prime}}, \ldots, \partial_{n}^{\prime}=\frac{\partial}{\partial x_{n}^{\prime}} .
$$

Every derivation $\partial \in E_{n}$ is a unique sum $\partial=\sum_{i=1}^{n} a_{i} \partial_{i}$ where $a_{i}=\partial * x_{i} \in Q_{n}$. Let $\partial:=$ $\left(\partial_{1}, \ldots, \partial_{n}\right)^{T}$ and $\partial^{\prime}:=\left(\partial_{1}^{\prime}, \ldots, \partial_{n}^{\prime}\right)^{T}$ where $T$ stands for the transposition. Then

$$
\partial^{\prime}=J(\sigma)^{-1} \partial, \text { i.e. } \partial_{i}^{\prime}=\sum_{j=1}^{n}\left(J(\sigma)^{-1}\right)_{i j} \partial_{j} \text { for } i=1, \ldots, n \text {. }
$$

In more detail, if $\partial^{\prime}=A \partial$ where $A=\left(a_{i j}\right) \in M_{n}\left(Q_{n}\right)$, i.e. $\partial_{i}=\sum_{j=1}^{n} a_{i j} \partial_{j}$. Then for all $i, j=1, \ldots, n$,

$$
\delta_{i j}=\partial_{i}^{\prime} * x_{j}^{\prime}=\sum_{k=1}^{n} a_{i k} \frac{\partial x_{j}^{\prime}}{\partial x_{k}}
$$


where $\delta_{i j}$ is the Kronecker delta function. The equalities above can be written in the matrix form as $A J(\sigma)=1$ where 1 is the identity matrix. Therefore, $A=J(\sigma)^{-1}$.

The maximal abelian Lie subalgebra $\mathcal{D}_{n}$ of $E_{n}$. Suppose that a group $G$ acts on a set $S$. For a nonempty subset $T$ of $S, \mathrm{St}_{G}(T):=\{g \in G \mid g T=T\}$ is the stabilizer of the set $T$ in $G$ and $\operatorname{Fix}_{G}(T):=\{g \in G \mid g t=t$ for all $t \in T\}$ is the fixator of the set $T$ in $G$. Clearly, $\operatorname{Fix}_{G}(T)$ is a normal subgroup of $\mathrm{St}_{G}(T)$.

Lemma 2.4 1. $C_{E_{n}}\left(\mathcal{D}_{n}\right)=\mathcal{D}_{n}$ and so $\mathcal{D}_{n}$ is a maximal abelian Lie subalgebra of $E_{n}$.

$$
\begin{aligned}
& \text { 2. } \operatorname{Fix}_{\mathbb{Q}_{n}}\left(\mathcal{D}_{n}\right)=\operatorname{Fix}_{\mathbb{Q}_{n}}\left(\partial_{1}, \ldots, \partial_{n}\right)=\operatorname{Sh}_{n} . \\
& \text { 3. } \operatorname{Fix}_{\mathbb{Q}_{n}}=\left(\partial_{1}, \ldots, \partial_{n}, H_{1}, \ldots, H_{n}\right)=\{e\} . \\
& \text { 4. } \operatorname{Cen}_{E_{n}}\left(\mathcal{D}_{n}+\mathcal{H}_{n}\right)=0 .
\end{aligned}
$$

Proof. 1. Statement 1 follows from (2): Clearly, $\mathcal{D}_{n} \subseteq C_{E_{n}}\left(\mathcal{D}_{n}\right)$. Let $\partial=\sum a_{i} \partial_{i} \in C_{E_{n}}\left(\mathcal{D}_{n}\right)$ where $a_{i} \in Q_{n}$. Then all elements $a_{i} \in \bigcap_{i=1}^{n} \operatorname{ker}_{Q_{n}} \partial_{i}=K$, by (21), and so $\partial \in \mathcal{D}_{n}$. So, $C_{E_{n}}\left(\mathcal{D}_{n}\right)=$ $\mathcal{D}_{n}$ and as a result $\mathcal{D}_{n}$ is a maximal abelian Lie subalgebra of $E_{n}$.

2. Let $\sigma \in \operatorname{Fix}_{\mathbb{Q}_{n}}\left(\mathcal{D}_{n}\right)$ and $J(\sigma)=\left(J_{i j}\right)$. By (5) $), \partial=J(\sigma) \partial$, and so, for all $i, j=1, \ldots, n$, $\delta_{i j}=\partial_{i} * x_{j}=J_{i j}$, i.e. $J(\sigma)=1$, or equivalently, by (2),

$$
x_{1}^{\prime}=x_{1}+\lambda_{1}, \ldots, x_{n}^{\prime}=x_{n}+\lambda_{n}
$$

for some scalars $\lambda_{i} \in K$, and so $\sigma \in \operatorname{Sh}_{n}$ (since $x_{i}^{\prime}-x_{i} \in \bigcap_{j=1}^{n} \operatorname{ker}_{Q_{n}}\left(\partial_{j}\right)=K$ for $\left.i=1, \ldots, n\right)$.

3. Let $\sigma \in \operatorname{Fix}_{\mathbb{Q}_{n}}=\left(\partial_{1}, \ldots, \partial_{n}, H_{1}, \ldots, H_{n}\right)$. Then $\sigma \in \operatorname{Fix}_{\mathbb{Q}_{n}}\left(\partial_{1}, \ldots, \partial_{n}\right)=\mathrm{Sh}_{n}$, by statement 2. So, $\sigma\left(x_{1}\right)=x_{1}+\lambda_{1}, \ldots, \sigma\left(x_{n}\right)=x_{n}+\lambda_{n}$ where $\lambda_{i} \in K$. Then $x_{i} \partial_{i}=\sigma\left(x_{i} \partial_{i}\right)=\left(x_{i}+\right.$ $\left.\lambda_{i}\right) \partial_{i}$ for $i=1, \ldots, n$, and so $\lambda_{1}=\cdots=\lambda_{n}=0$. This means that $\sigma=e$. So, Fix $_{\mathbb{Q}_{n}}=$ $\left(\partial_{1}, \ldots, \partial_{n}, H_{1}, \ldots, H_{n}\right)=\{e\}$.

4. Statement 4 follows from statement 1 and Lemma 2.2,

Lemma 2.5 Let $A$ be a $K$-algebra, $\operatorname{Der}_{K}(A)$ be the Lie algebra of $K$-derivations of $A$ and $\mathcal{D}(A)$ be the ring of differential operators on $A$. If the algebra $\mathcal{D}(A)$ is simple and generated by $A$ and $\operatorname{Der}_{K}(A)$ then the $\mathcal{D}(A)$-module $A$ is simple.

Proof. Let $\mathfrak{a}$ be a nonzero $\mathcal{D}(A)$-submodule of $A$. So, $\mathfrak{a}$ is an ideal of $A$ such that $\partial(\mathfrak{a}) \subseteq \mathfrak{a}$ for all $\partial \in \operatorname{Der}_{K}(A)$. The algebra $\mathcal{D}:=\mathcal{D}(A)$ is generated by $A$ and $D$. So, $\mathcal{D} \mathfrak{a} \subseteq \mathfrak{a} \mathcal{D}$ and $\mathfrak{a} \mathcal{D} \subseteq \mathcal{D} \mathfrak{a}$, i.e. $\mathcal{D} \mathfrak{a}=\mathfrak{a} \mathcal{D}$ is a nonzero ideal of the simple algebra $\mathcal{D}$. Hence, $1 \in \mathcal{D} \mathfrak{a}$ and so $1=\sum_{i} a_{i} d_{i}$ for some elements $d_{i} \in \mathcal{D}$ and $a_{i} \in \mathfrak{a} \subseteq D$. Then

$$
1=1 * 1=\sum_{i} a_{i} d_{i} * 1 \in \mathfrak{a},
$$

hence $\mathfrak{a}=A$, i.e. $A$ is a simple $\mathcal{D}(A)$-module.

Theorem 2.6 $\quad$ 1. $E_{n}$ is a simple Lie algebra.

2. $Z\left(E_{n}\right)=\{0\}$.

3. $\left[E_{n}, E_{n}\right]=E_{n}$.

Proof. 1. (i) $n=1$, i.e. $E_{1}=K(x) \partial$ is a simple Lie algebra: We split the proof into several steps.

(a) $D_{1}:=K[x] \partial$ and $W_{1}:=K\left[x, x^{-1}\right] \partial$ are simple Lie subalgebras of $E_{1}$ (easy).

(b) For all $\lambda \in K, W_{1}(\lambda):=K\left[x,(x-\lambda)^{-1}\right]$ is a simple Lie subalgebra of $E_{1}$, by applying the $K$-automorphism $s_{\lambda}: x \mapsto x-\lambda$ of the $K$-algebra $Q_{1}$ to $W_{1}$, i.e. $s_{\lambda}\left(W_{1}\right)=W_{1}(\lambda)$.

(c) For any nonempty subset $I \subset K, W_{1}(I):=W_{1}(I)_{K}:=K\left[x,(x-\lambda)^{-1} \mid \lambda \in I\right] \partial$ is a simple Lie subalgebra of $E_{1}$ : Let $\mathfrak{a}$ be a nonzero ideal of $W_{1}(I)$ and $0 \neq a \partial \in \mathfrak{a}$. Then either $a \partial \in D_{1}$ or 
$0 \neq[p \partial, a \partial] \in D_{1} \cap \mathfrak{a}$ for some $p \in P_{1}$. Since $D_{1} \subseteq W_{1}(\lambda)$ for all $\lambda \in I$ and $W_{1}(\lambda)$ are simple Lie algebra, $\mathfrak{a} \cap W_{1}(\lambda)=W_{1}(\lambda)$. Hence $\mathfrak{a}=W_{1}(I)$ since

$$
W_{1}(I)=\bigcup_{\lambda \in I} W_{1}(\lambda)
$$

i.e. $W_{1}(I)$ is a simple Lie algebra.

(d) If $K$ is an algebraically closed field then $E_{1}$ is a simple Lie algebra since $E_{1}=W_{1}(K)$.

The algebra $E_{1}$ is the union $\bigcup_{0 \neq f \in P_{1}} W_{1}\left[f^{-1}\right]$ of the Lie algebras $W_{1}\left[f^{-1}\right]:=P_{1, f} \partial$ where $P_{1, f}$ is the localization of $P_{1}$ at the powers of the element $f$. Let $\mathfrak{a}$ be the ideal of $E_{1}$ generated by a nonzero element $a=p q^{-1} \partial$ for some $p q^{-1} \in Q_{1}$. Clearly, $a \in W_{1}\left[(f q)^{-1}\right]$ for all nonzero elements $f \in P_{1}$ and $E_{1}=\bigcup_{0 \neq f \in P_{1}} W_{1}\left[(f g)^{-1}\right]$. So, to finish the proof of (i) it suffices to show that all the algebras $W_{1}\left[f^{-1}\right]$ are simple.

(e) $A:=W_{1}\left[f^{-1}\right]$ is a simple Lie algebra for all $0 \neq f \in P_{1}$ : Let $K^{\prime}:=K\left(\nu_{1}, \ldots, \nu_{s}\right)$ be the subfield of the algebraic closure $\bar{K}$ of $K$ generated by the roots $\nu_{1}, \ldots, \nu_{s}$ of the polynomial $f$ and $G=\operatorname{Gal}\left(K^{\prime} / K\right)$ be the Galois group of the finite Galois field extension $K^{\prime} / K$ (since char $\left.(K)=0\right)$. Let $K^{\prime}=\oplus_{i=1}^{d} K \theta_{i}$ for some elements $\theta_{i} \in K^{\prime}$ and $\theta_{1}=1$. By (c),

$$
A^{\prime}:=K^{\prime}\left[x, f^{-1}\right] \partial=W_{1}\left(\nu_{1}, \ldots, \nu_{s}\right)_{K^{\prime}}
$$

is a simple Lie $K^{\prime}$-algebra. Let $a \in A \backslash\{0\}, \mathfrak{a}$ and $\mathrm{d} \mathfrak{a}^{\prime}$ be the ideals in $A$ and $A^{\prime}$ respectively that are generated by the element $a$. Then $\mathfrak{a}^{\prime}=A^{\prime}$, by (c). Notice that $A^{\prime}=\sum_{i=1}^{d} \theta_{i} A$ and for $a^{\prime}=\sum_{i=1}^{d} \theta_{i} a_{i}, b=\sum_{i=1}^{d} \theta_{i} b_{i} \in A^{\prime}$ where $a_{i}, b_{i} \in A,\left[a^{\prime}, b\right]=\sum_{i=1}^{d} \theta_{i} \theta_{j}\left[a_{i}, b_{j}\right]$. Moreover, every element in $A^{\prime}=\mathfrak{a}^{\prime}$ is a linear combination of several commutators in $A^{\prime}$ (where $c=\sum_{i=1}^{d} \theta_{k} c_{k} \in A^{\prime}$ and $c_{k} \in A$ ),

$$
\left[a,\left[a^{\prime}, \ldots[b, c] \ldots\right]=\sum \theta_{i} \cdots \theta_{j} \theta_{k}\left[a,\left[a_{i}, \ldots\left[b_{j}, c_{k}\right] \ldots\right] .\right.\right.
$$

The symmetrization map Sym : $K^{\prime} \rightarrow K, \lambda \mapsto|G|^{-1} \sum_{g \in G} g(\lambda)$, is a surjection such that $\operatorname{Sym}(\mu)=\mu$ for all $\mu \in K$. Clearly, $K^{\prime}(x) / K(x)$ is a Galois field extension with the Galois group $G$ where the elements of $G$ act trivially on the element $x$. So, the symmetrization map Sym can be extended to the surjection $K^{\prime}(x) \rightarrow K(x)$ by the same rule, and then to the surjection $A^{\prime} \rightarrow A, f \partial \mapsto \operatorname{Sym}(f) \partial$.

Each element $e \in A \subseteq A^{\prime}$, can be expressed as a finite sum of elements in (6). Then applying Sym, we see that $e$ is a linear combination of elements (commutators) from $\mathfrak{a}$, i.e. $A$ is a simple Lie algebra.

(ii) $E_{n}$ is a simple Lie algebra for $n \geq 2$ : Let $a \in E_{n} \backslash\{0\}$ and $\mathfrak{a}=(a)$ be the ideal in $E_{n}$ generated by the element $a=\sum_{i=1}^{n} a_{i} \partial_{i}$ where $a_{i} \in Q_{n}$.

(a) $\mathfrak{a} \cap D_{n} \neq 0$ : If $a \in D_{n}$ then there is nothing to prove. Suppose that $a \notin D_{n}$.

(a1) Suppose that $a_{i} \in K\left(x_{i}\right)$ for all $i$. Then $a_{i} \notin K\left[x_{i}\right]$ for some $i$ (since $a \notin D_{n}$ ), and so

$$
\mathfrak{a} \ni\left[H_{i}, a\right]=H_{i}\left(a_{i}\right) \partial_{i} \in K\left(x_{i}\right) \partial_{i} \backslash\{0\} .
$$

By (i), $\partial_{1} \in \mathfrak{a} \cap D_{n}$.

(a2) Suppose that $a_{i} \notin K\left(x_{i}\right)$ for some $i$. Then $\partial_{j}\left(a_{i}\right) \neq 0$ for some $j \neq i$. Let $q \in P_{n}$ be the common denominator of the fractions $a_{1}, \ldots, a_{n}$, that is $a_{1}=p_{1} q^{-1}, \ldots, a_{n}=p_{n} q^{-1}$ for some elements $p_{i} \in P_{n}$. For all $n \geq 2$,

$$
D_{n} \cap \mathfrak{a} \ni\left[q^{n} \partial_{j}, a\right]=q^{n} \partial_{j}\left(a_{i}\right) \partial_{i}+\sum_{k \neq i}(\ldots) \partial_{k} \neq 0 .
$$

(b) $\mathfrak{a}=D_{n}$ since $D_{n}$ is a simple Lie algebra, 4.

(c) $\mathfrak{a} \supseteq K\left(x_{i}\right) \partial_{i}$ for $i=1, \ldots, n$ : In view of symmetry it suffices to prove that $\mathfrak{a} \supseteq K\left(x_{1}\right) \partial_{1}$. Notice that for all $u \in Q_{n}$ and $i=2, \ldots, n$,

$$
\mathfrak{a} \ni\left[u \partial_{1}, x_{1} \partial_{i}\right]=u \partial_{i}-x_{1} \partial_{i}(u) \partial_{1} .
$$


Therefore, $\mathfrak{a}+Q_{n} \partial_{1}=E_{n}$. The field of rational functions $Q_{n}=Q_{n}(K)$ can be seen as the field of rational functions $Q_{n}(K)=Q_{n-1}\left(K^{\prime}\right)$ where $K^{\prime}=K\left(x_{1}\right)$. Then

$$
E_{n-1}^{\prime}:=\operatorname{Der}_{K^{\prime}}\left(Q_{n-1}\left(K^{\prime}\right)\right)=\bigoplus_{i=2}^{n} Q_{n-1}\left(K^{\prime}\right) \partial_{i}=\bigoplus_{i=2}^{n} Q_{n} \partial_{i}
$$

By Lemma 2.5 the $E_{n-1}^{\prime}$-module $Q_{n-1}^{\prime} / K^{\prime}=Q_{n} / K\left(x_{1}\right)$ is simple. The Lie algebra $E_{n-1}^{\prime}$ is a Lie subalgebra of $E_{n}$, and $E_{n}$ can be seen as a left $E_{n-1}^{\prime}$-module with respect to the adjoint action. The ideal $\mathfrak{a}$ of $E_{n}$ is an $E_{n-1}^{\prime}$-submodule of $E_{n}$. The Lie algebra $K\left(x_{1}\right) \partial_{1}$ is simple and $\mathfrak{a} \cap K\left(x_{1}\right) \partial_{1}$ is a nonzero ideal of it (by (b)). Therefore, $K\left(x_{1}\right) \partial_{1} \subseteq \mathfrak{a}$. The $E_{n-1}^{\prime}$-module $E_{n} / \mathfrak{a}=\left(\mathfrak{a}+Q_{n} \partial_{1}\right) / \mathfrak{a} \simeq Q_{n} \partial_{1} / \mathfrak{a} \cap Q_{n} \partial_{1}$ is an epimorphic image of the simple $E_{n-1}^{\prime}$-module $Q_{n} / K\left(x_{1}\right)$ via

$$
\varphi: Q_{n} / K\left(x_{1}\right) \rightarrow Q_{n} \partial_{1} / \mathfrak{a} \cap Q_{n} \partial_{1}, \quad u+K\left(x_{1}\right) \mapsto u \partial_{1}+\mathfrak{a} \cap Q_{n} \partial_{1},
$$

with $0 \neq\left(P_{n}+K\left(x_{1}\right)\right) / K\left(x_{1}\right) \subseteq \operatorname{ker}(\varphi)$. Therefore, $Q_{n} \partial_{1}=\mathfrak{a} \cap Q_{n} \partial_{1} \subseteq \mathfrak{a}$, and so $E_{n}=\mathfrak{a}+Q_{n} \partial_{1}=$ $\mathfrak{a}$. So, $E_{n}$ is a simple Lie algebra.

2 and 3 . Statements 2 and 3 follow from statement 1.

Lemma 2.7 For all nonzero elements $q \in Q_{n}$ and $i=1, \ldots, n, C_{E_{n}}\left(q P_{n} \partial_{i}\right)=\{0\}$.

Proof. Let $c \in C_{E_{n}}\left(q P_{n} \partial_{i}\right)$. Then for all elements $p \in P_{n}$,

$$
0=\left[c, q p \partial_{i}\right]=c(p) \cdot q \partial_{i}+p\left[c, q \partial_{i}\right]=c(p) \cdot q \partial_{i} .
$$

Then $c(p)=0$ for all $p \in P_{n}$, and so $c=0$.

Proposition 2.8 [4] $\operatorname{Fix}_{\mathbb{G}_{n}}\left(\partial_{1}, \ldots, \partial_{n}, H_{1}, \ldots, H_{n}\right)=\{e\}$.

Let $d_{1}, \ldots, d_{n}$ be a commuting linear maps acting in a vector space $E$. Let $\operatorname{Nil}_{E}\left(d_{1}, \ldots, d_{n}\right):=$ $\left\{e \in E \mid d_{i}^{j} e=0\right.$ for all $i=1, \ldots, n$ and some $\left.j=j(e)\right\}$. Let $\operatorname{Nil}_{E_{n}}\left(\mathcal{D}_{n}\right):=\operatorname{Nil}_{E_{n}}\left(\delta_{1}, \ldots, \delta_{n}\right)$. Clearly, $\operatorname{Nil}_{E_{n}}\left(\mathcal{D}_{n}\right)=D_{n}$ is a Lie subalgebra of $E_{n}$.

Proposition 2.9 1. $\operatorname{Fix}_{\mathbb{E}_{n}}\left(\partial_{1}, \ldots, \partial_{n}, H_{1}, \ldots, H_{n}\right)=\{e\}$.

2. $\operatorname{Fix}_{\mathbb{E}_{n}}\left(\partial_{1}, \ldots, \partial_{n}\right)=\mathrm{Sh}_{n}$.

Proof. 1. Let $\sigma \in F:=\operatorname{Fix}_{\mathbb{E}_{n}}\left(\partial_{1}, \ldots, \partial_{n}, H_{1}, \ldots, H_{n}\right)$. We have to show that $\sigma=e$. Then $\sigma^{-1} \in F$ and $\sigma^{ \pm 1}\left(\operatorname{Nil}_{E_{n}}\left(\mathcal{D}_{n}\right)\right) \subseteq \operatorname{Nil}_{E_{n}}\left(\mathcal{D}_{n}\right)$, i.e. $\sigma\left(D_{n}\right)=D_{n}$ since $\mathrm{Nil}_{E_{n}}\left(\mathcal{D}_{n}\right)=D_{n}$. So, $\left.\sigma\right|_{D_{n}} \in \operatorname{Fix}_{\mathbb{G}_{n}}\left(\partial_{1}, \ldots, \partial_{n}, H_{1}, \ldots, H_{n}\right)=\{e\}$ (Proposition [2.8), i.e. $\sigma(\partial)=\partial$ for all $\partial \in D_{n}$. Let $0 \neq \delta \in E_{n}$. Then $\delta=q^{-1} \partial$ for some $0 \neq q \in P_{n}$ and $\partial \in D_{n}$. Now, $\left[q^{2} p \partial_{i}, \delta\right]=\partial^{\prime} \in D_{n}$ for all $p \in P_{n}$. Applying $\sigma$ to the equality yields the equality $\left[q^{2} p \partial_{i}, \sigma(\delta)\right]=\partial^{\prime}$. By taking the difference, we obtain $\sigma(\delta)-\delta \in C_{E_{n}}\left(q^{2} P_{n} \partial_{i}\right)=\{0\}$, by Lemma 2.7 hence $\sigma=e$.

2. Clearly, $\mathrm{Sh}_{n} \subseteq F:=\mathrm{Fix}_{\mathbb{E}_{n}}\left(\partial_{1}, \ldots, \partial_{n}\right)$. Let $\sigma \in F$ and $H_{i}^{\prime}:=\sigma\left(H_{i}\right), \ldots, H_{n}^{\prime}:=\sigma\left(H_{n}\right)$. Applying the automorphism $\sigma$ to the commutation relations $\left[\partial_{i}, H_{j}\right]=\delta_{i j} \partial_{i}$ gives the relations $\left[\partial_{i}, H_{j}^{\prime}\right]=\delta_{i j} \partial_{i}$. By taking the difference, we see that $\left[\partial_{i}, H_{j}^{\prime}-H_{j}\right]=0$ for all $i$ and $j$. Therefore, $H_{i}^{\prime}=H_{i}+d_{i}$ for some elements $d_{i} \in C_{E_{n}}\left(\mathcal{D}_{n}\right)=\mathcal{D}_{n}$ (Lemma 2.4 (1)), and so $d_{i}=\sum_{j=1}^{n} \lambda_{i j} \partial_{j}$ for some elements $\lambda_{i j} \in K$. The elements $H_{1}^{\prime}, \ldots, H_{n}^{\prime}$ commute, hence

$$
\left[H_{j}, d_{i}\right]=\left[H_{i}, d_{j}\right] \text { for all } i, j,
$$

or equivalently,

$$
\lambda_{i j} \partial_{j}=\lambda_{j i} \partial_{i} \text { for all } i, j .
$$

This means that $\lambda_{i j}=0$ for all $i \neq j$, i.e.

$$
H_{i}^{\prime}=H_{i}+\lambda_{i i} \partial_{i}=\left(x_{i}+\lambda_{i i}\right) \partial_{i}=s_{\lambda}\left(H_{i}\right)
$$


where $s_{\lambda} \in \mathrm{Sh}_{n}, s_{\lambda}\left(x_{i}\right)=x_{i}+\lambda_{i i}$ for all $i$. Then $s_{\lambda}^{-1} \sigma \in \operatorname{Fix}_{\mathbb{E}_{n}}\left(\partial_{1}, \ldots, \partial_{n}, H_{1}, \ldots, H_{n}\right)=\{e\}$ (statement 1), and so $\sigma=s_{\lambda} \in \mathrm{Sh}_{n}$.

The automorphism $\nu$. Let $\nu$ be the $K$-automorphism of $Q_{n}$ given by the rule $\nu\left(x_{i}\right)=x_{i}^{-1}$ for $i=1, \ldots, n$. Then

$$
\nu\left(\partial_{i}\right)=-x_{i} H_{i}, \quad \nu\left(H_{i}\right)=-H_{i}, \quad \nu\left(x_{i} H_{i}\right)=-\partial_{i}, \quad i=1, \ldots, n .
$$

By (7), the elements $X_{1}:=x_{1} H_{1}, \ldots, X_{n}:=x_{n} H_{n}$ commute and the next lemma follows from Lemma 2.4 and Proposition 2.9 since $\mathcal{X}_{n}:=\nu\left(\mathcal{D}_{n}\right)=\bigoplus_{i=1}^{n} K X_{i}$.

Lemma 2.10 1. $C_{E_{n}}\left(\mathcal{X}_{n}\right)=\mathcal{X}_{n}$ is a maximal abelian Lie subalgebra of $E_{n}$.

2. $\operatorname{Fix}_{\mathbb{Q}_{n}}\left(X_{1}, \ldots, X_{n}\right)=\operatorname{Fix}_{\mathbb{E}_{n}}\left(X_{1}, \ldots, X_{n}\right)=\operatorname{Sh}_{n}$.

3. $\operatorname{Fix}_{\mathbb{Q}_{n}}\left(X_{1}, \ldots, X_{n}, H_{1}, \ldots, H_{n}\right)=\operatorname{Fix}_{\mathbb{E}_{n}}\left(X_{1}, \ldots, X_{n}, H_{1}, \ldots, H_{n}\right)=\{e\}$.

The following lemma is well-known and it is easy to prove.

Lemma 2.11 Let $\partial$ be a locally nilpotent derivation of a commutative $K$-algebra $A$ such that $\partial(x)=1$ for some element $x \in A$. Then $A=A^{\partial}[x]$ is a polynomial algebra over the ring $A^{\partial}:=\operatorname{ker}(\partial)$ of constants of the derivation $\partial$ in the variable $x$.

The next lemma is the core of the proof of Theorem 1.2 ,

Lemma 2.12 Let $\sigma \in \mathbb{E}_{n}, \partial_{1}^{\prime}:=\sigma\left(\partial_{1}\right), \ldots, \partial_{n}^{\prime}:=\sigma\left(\partial_{n}\right)$ and $\delta_{1}^{\prime}:=\operatorname{ad}\left(\partial_{1}^{\prime}\right), \ldots, \delta_{n}^{\prime}:=\operatorname{ad}\left(\partial_{n}^{\prime}\right)$. Then

1. $\partial_{1}^{\prime}, \ldots, \partial_{n}^{\prime}$ are commuting derivations of $Q_{n}$ such that $\bigcap_{i=1}^{n} \operatorname{ker}_{Q_{n}}\left(\partial_{i}^{\prime}\right)=K$.

2. $E_{n}=\bigoplus_{i=1}^{n} Q_{n} \partial_{i}^{\prime}$.

3. For each $i=1, \ldots, n, \sigma\left(x_{i} \partial_{i}\right)=x_{i}^{\prime} \partial_{i}^{\prime}$ for some elements $x_{i}^{\prime} \in Q_{n}$. The elements $x_{1}^{\prime}, \ldots, x_{n}^{\prime}$ are algebraically independent and $\partial_{i}^{\prime}\left(x_{j}^{\prime}\right)=\delta_{i j}$ for $i, j=1, \ldots, n$.

4. $\operatorname{Nil}_{Q_{n}}\left(\partial_{1}^{\prime}, \ldots, \partial_{n}^{\prime}\right)=P_{n}^{\prime}$ where $P_{n}^{\prime}:=K\left[x_{1}^{\prime}, \ldots, x_{n}^{\prime}\right]$.

5. $\operatorname{Nil}_{E_{n}}\left(\delta_{1}^{\prime}, \ldots, \delta_{n}^{\prime}\right)=\bigoplus_{i=1}^{n} P_{n}^{\prime} \partial_{i}^{\prime}$.

6. $\sigma\left(x^{\alpha} \partial_{i}\right)=x^{\prime \alpha} \partial_{i}^{\prime}$ for all $\alpha \in \mathbb{N}^{n}$ and $i=1, \ldots, n$.

7. $\sigma^{\prime}: Q_{n} \rightarrow Q_{n}, x_{i} \mapsto x_{i}^{\prime}, i=1, \ldots, n$ is a $K$-algebra homomorphism (statement 3) such that $\sigma^{\prime}\left(a \partial_{i}\right)=\sigma^{\prime}(a) \sigma\left(\partial_{i}\right)$.

8. The K-algebra homomorphism $\sigma^{\prime}$ is an automorphism.

Proof. 1. The elements $\partial_{1}, \ldots, \partial_{n}$ are commuting derivations, hence so are $\partial_{1}^{\prime}, \ldots, \partial_{n}^{\prime}$. Let $\lambda \in \bigcap_{i=1}^{n} \operatorname{ker}_{Q_{n}}\left(\partial_{i}^{\prime}\right)$. Then

$$
\lambda \partial_{1}^{\prime} \in C_{E_{n}}\left(\partial_{1}^{\prime}, \ldots, \partial_{n}^{\prime}\right)=\sigma\left(C_{E_{n}}\left(\partial_{1}, \ldots, \partial_{n}\right)\right)=\sigma\left(C_{E_{n}}\left(\mathcal{D}_{n}\right)\right)=\sigma\left(\mathcal{D}_{n}\right)=\sigma\left(\bigoplus_{i=1}^{n} K \partial_{i}\right)=\bigoplus_{i=1}^{n} K \partial_{i}^{\prime},
$$

since $C_{E_{n}}\left(\mathcal{D}_{n}\right)=\mathcal{D}_{n}$, Lemma 2.4.(1). Then $\lambda \in K$ since otherwise the infinite dimensional space $\bigoplus_{i>0} K \lambda^{i} \partial_{1}^{\prime}$ would be a subspace of the finite dimensional space $\sigma\left(\mathcal{D}_{n}\right)$.

2. It suffices to show that the elements $\partial_{1}^{\prime}, \ldots, \partial_{n}^{\prime}$ of the $n$-dimensional (left) vector space $E_{n}$ over the field $Q_{n}$ are $Q_{n}$-linearly independent (the key reason for that is statement 1). Let $V=\sum_{i=1}^{n} Q_{n} \partial_{i}^{\prime}$. Suppose that $m:=\operatorname{dim}_{Q_{n}}(V)<n$, we seek a contradiction. Up to order, let $\partial_{1}^{\prime}, \ldots, \partial_{m}^{\prime}$ be a $Q_{n}$-basis of $V$. Then $\partial_{m+1}=\sum_{i=1}^{m} a_{i} \partial_{i}^{\prime}$ for some elements $a_{i} \in Q_{n}$. By applying $\delta_{j}^{\prime}(j=1, \ldots, n)$, we see that $0=\sum_{i=1}^{m} \partial_{j}^{\prime}(a) \partial_{i}^{\prime}$, and so $a_{i} \in \bigcap_{i=1}^{n} \operatorname{ker}_{Q_{n}}\left(\partial_{j}^{\prime}\right)=K$, by statement 1 . This means that the elements $\partial_{1}^{\prime}, \ldots, \partial_{m}^{\prime}$ are $K$-linearly dependent, a contradiction. 
3. Let $H_{i}^{\prime}:=\sigma\left(x_{i} \partial_{i}\right)$ for $i=1, \ldots, n$. By statement $2, H_{i}^{\prime}=\sum_{i=1}^{n} a_{i j} \partial_{j}^{\prime}$ for some elements $a_{i j} \in Q_{n}$. Applying the automorphism $\sigma$ to the relations $\delta_{i j} \partial_{j}=\left[\partial_{j}, H_{i}\right]$ yields the relations

$$
\delta_{i j} \partial_{i}^{\prime}=\sum_{i=1}^{n} \partial_{j}^{\prime}\left(a_{i k}\right) \partial_{k}^{\prime}
$$

Let $x_{i}^{\prime}:=a_{i i}$. Then $\partial_{j}^{\prime}\left(x_{i}^{\prime}\right)=\delta_{j i}$ and $\partial_{j}^{\prime}\left(a_{i k}\right)=0$ for all $k \neq i$. By statement $1, a_{i k} \in K$ for all $i \neq k$. Now,

$$
H_{i}^{\prime}:=x_{i}^{\prime} \partial_{i}^{\prime}+\sum_{j \neq i} a_{i j} \partial_{j}^{\prime} .
$$

The elements $H_{1}^{\prime}, \ldots, H_{n}^{\prime}$ commute, hence for all $i \neq j, 0=\left[H_{i}^{\prime}, H_{j}^{\prime}\right]=-a_{j i} \partial_{i}^{\prime}+a_{i j} \partial_{j}^{\prime}$, and so $a_{i j}=0$. Therefore, $H_{i}^{\prime}=x_{i}^{\prime} \partial_{i}^{\prime}$.

The equalities $\partial_{i}^{\prime}\left(x_{j}^{\prime}\right)=\delta_{i j}$ imply that the elements $x_{1}^{\prime}, \ldots, x_{n}^{\prime} \in Q_{n}$ are algebraically independent over $K$ : Suppose that $f\left(x_{1}^{\prime}, \ldots, x_{n}^{\prime}\right)=0$ for some nonzero polynomial $f\left(t_{1}, \ldots, x_{n}\right) \in$ $K\left[t_{1}, \ldots, x_{n}\right]$. We can assume that the (total) degree $\operatorname{deg}(f)$ is the least possible. Clearly, $f \notin K$, hence $\frac{\partial f}{\partial x_{i}} \neq 0$ for some $i$ and $\operatorname{deg}\left(\frac{\partial f}{\partial x_{i}}\right)<\operatorname{deg}(f)$, but $\frac{\partial f}{\partial x_{i}}\left(x_{1}^{\prime}, \ldots, x_{n}^{\prime}\right)=\partial_{i}\left(f\left(x_{1}^{\prime}, \ldots, x_{n}^{\prime}\right)\right)=$ $\partial_{i}(0)=0$, a contradiction.

4. Let $\mathcal{D}_{n}^{\prime}=\sum_{i=1}^{n} K \partial_{i}^{\prime}$ and $N=\operatorname{Nil}_{Q_{n}}\left(\mathcal{D}_{n}^{\prime}\right)$. By statement 3 and Lemma 2.11,

$$
N=N^{\mathcal{D}_{n}^{\prime}}\left[x_{1}^{\prime}, \ldots, x_{n}^{\prime}\right]=K\left[x_{1}^{\prime}, \ldots, x_{n}^{\prime}\right]
$$

since $K \subseteq N^{\mathcal{D}_{n}^{\prime}} \subseteq Q_{n}^{\mathcal{D}_{n}^{\prime}}=K$ (by statement 1 ).

5. Let $\partial=\sum_{i=1}^{n} a_{i} \partial_{i}^{\prime} \in N:=\operatorname{Nil}_{E_{n}}\left(\delta_{1}^{\prime}, \ldots, \delta_{n}^{\prime}\right)$ where $a_{i} \in Q_{n}$ (statement 2). For all $\alpha \in \mathbb{N}^{n}$,

$$
\delta^{\prime \alpha}(\partial)=\sum_{i=1}^{n} \partial^{\prime \alpha}\left(a_{i}\right) \partial_{i}^{\prime}
$$

where $\delta^{\prime \alpha}:=\prod_{i=1}^{n} \delta_{i}^{\prime \alpha_{i}}, \delta_{i}^{\prime}=\operatorname{ad}\left(\partial_{i}^{\prime}\right)$ and $\partial^{\prime \alpha}:=\prod_{i=1}^{n} \partial_{i}^{\prime \alpha_{i}}$. So, $\delta^{\prime \alpha}\left(a_{i}\right)=0$ iff $\partial^{\prime \alpha}\left(a_{i}\right)=0$ for $i=1, \ldots, n$ (statement 2). Now, statement 5 follows from statement 4 .

6. First, let us show that, by induction on $|\alpha|$, that $\sigma\left(x^{\alpha} \partial_{i}\right)-x^{\prime \alpha} \partial_{i}^{\prime} \in \operatorname{Cen}_{E_{n}}\left(\mathcal{D}_{n}^{\prime}\right)=\mathcal{D}_{n}^{\prime}$ (Lemma 2.4.(1)). The initial case when $|\alpha|=0$ is obvious. So, let $|\alpha|>0$. Then

$$
\begin{aligned}
{\left[\partial_{j}^{\prime}, \sigma\left(x^{\alpha} \partial_{i}\right)-x^{\alpha \alpha} \partial_{i}^{\prime}\right] } & =\sigma\left(\left[\partial_{j}, x^{\alpha} \partial_{i}\right]\right)-\alpha_{j} x^{\prime \alpha-e_{j}} \partial_{i}^{\prime}=\sigma\left(\alpha_{j} x^{\alpha-e_{j}} \partial_{i}\right)-\alpha_{j} x^{\prime \alpha-e_{j}} \partial_{i}^{\prime} \\
& =\alpha_{j} x^{\prime \alpha-e_{j}} \partial_{i}^{\prime}-\alpha_{j} x^{\prime \alpha-e_{j}} \partial_{i}^{\prime}=0 .
\end{aligned}
$$

Therefore, $\sigma\left(x^{\alpha} \partial_{i}\right)=x^{\prime \alpha} \partial_{i}^{\prime}+\sum \lambda_{i j} \partial_{j}^{\prime}$ for some scalars $\lambda_{i j}=\lambda_{i j}(\alpha) \in K$. Notice that

$$
\sigma\left(H_{i}\right)=\sigma\left(x_{i} \partial_{i}\right)=x_{i}^{\prime} \partial_{i}^{\prime}:=H_{i}^{\prime},
$$

by the definition of the elements $x_{i}^{\prime}$. Since $|\alpha|>0, \alpha_{j} \neq 0$ for some $j$. Applying the automorphism $\sigma$ to the equalities $\left(\alpha_{j}-\delta_{i j}\right) x^{\alpha} \partial_{i}=\left[H_{j}, x^{\alpha} \partial_{i}\right]$ we have (we may assume that $x^{\alpha} \partial_{i} \neq H_{i}$ )

$$
\begin{aligned}
\left(\alpha_{j}-\delta_{i j}\right)\left(x^{\prime \alpha} \partial_{i}^{\prime}+\sum_{k=1}^{n} \lambda_{i k} \partial_{k}^{\prime}\right) & =\sigma\left(\left(\alpha_{j}-\delta_{i j}\right) x^{\alpha} \partial_{i}\right)=\sigma\left(\left[H_{j}, x^{\alpha} \partial_{i}\right]\right)=\left[H_{j}^{\prime}, x^{\prime \alpha} \partial_{i}^{\prime}+\sum_{k=1}^{n} \lambda_{i k} \partial_{k}^{\prime}\right] \\
& =\left(\alpha_{j}-\delta_{i j}\right) x^{\prime \alpha} \partial_{i}^{\prime}-\lambda_{i j} \partial_{j}^{\prime},
\end{aligned}
$$

and so $\left(\alpha_{j}-\delta_{i j}+1\right) \lambda_{i j}=0$ and $\left(\alpha_{j}-\delta_{i j}\right) \lambda_{i k}=0$ for all $k \neq j$. This means that all $\lambda_{i s}=0$.

7. By statement $3, \sigma^{\prime}$ is a $K$-algebra homomorphism such that $\operatorname{im}\left(\sigma^{\prime}\right)=Q_{n}^{\prime}:=K\left(x_{1}^{\prime}, \ldots, x_{n}^{\prime}\right)$. By statement 3 , for all elements $a \in Q_{n}$,

$$
\partial_{i}^{\prime} \sigma^{\prime}(a)=\sigma^{\prime} \partial_{i}(a)
$$

since $\partial_{i}^{\prime}$ acts as $\frac{\partial}{\partial x_{i}^{\prime}}$ on $Q_{n}^{\prime}$. 
Let $a=p q^{-1} \neq 0$ where $p, q \in P_{n}$. Then, for all $r \in q^{2} P_{n},\left[a \partial_{i}, r \partial_{i}\right]=\left(a \partial_{i}(r)-\partial_{i}(a) r\right) \partial_{i} \in$ $P_{n} \partial_{i}$. By applying $\sigma$, we have the equality

$$
\left[\sigma\left(a \partial_{i}\right), \sigma^{\prime}(r) \partial_{i}^{\prime}\right]=\sigma^{\prime}\left(a \partial_{i}(r)-\partial_{i}(a) r\right) \partial_{i}^{\prime} .
$$

On the other hand,

$$
\begin{aligned}
{\left[\sigma^{\prime}(a) \partial_{i}^{\prime}, \sigma^{\prime}(r) \partial_{i}^{\prime}\right] } & =\left(\sigma^{\prime}(a) \partial_{i}^{\prime} \sigma^{\prime}(r)-\partial_{i}^{\prime} \sigma^{\prime}(a) \sigma^{\prime}(r)\right) \partial_{i}^{\prime}=\left(\sigma^{\prime}(a) \sigma^{\prime} \partial_{i}(r)-\sigma^{\prime} \partial_{i}(a) \sigma^{\prime}(r)\right) \partial_{i}^{\prime} \\
& =\sigma^{\prime}\left(a \partial_{i}(r)-\partial_{i}(a) r\right) \partial_{i}^{\prime} .
\end{aligned}
$$

Hence,

$\sigma\left(a \partial_{i}\right)-\sigma^{\prime}(a) \partial_{i}^{\prime} \in C_{E_{n}}\left(\sigma^{\prime}\left(q^{2} P_{n}\right) \partial_{i}^{\prime}\right)=C_{E_{n}}\left(\sigma\left(q^{2} P_{n} \partial_{i}\right)\right)=\sigma\left(C_{E_{n}}\left(q^{2} P_{n} \partial_{i}\right)\right)=\sigma\left(C_{E_{n}}\left(q^{2} P_{n} \partial_{i}\right)\right)=0$,

by Lemma 2.7. Therefore, $\sigma\left(a \partial_{i}\right)=\sigma^{\prime}(a) \sigma\left(\partial_{i}\right)$.

8. Since $\sigma\left(Q_{n} \partial_{i}\right)=\sigma^{\prime}\left(Q_{n}\right) \partial_{i}^{\prime}$ for all $i=1, \ldots, n$ (statement 7), we must have $\sigma^{\prime}\left(Q_{n}\right)=Q_{n}$, by statement 2 , and so $\sigma^{\prime} \in \mathbb{Q}_{n}$.

Proof of Theorem 1.2 Let $\sigma \in \mathbb{E}_{n}$. By Corollary 2.12. (8), we have the automorphism $\sigma^{\prime} \in$ $\mathbb{Q}_{n}$ such that, by Lemma $2.12,(3,6), \sigma^{\prime-1} \sigma \in \operatorname{Fix}_{\mathbb{E}_{n}}\left(\partial_{1}, \ldots, \partial_{n}, H_{1}, \ldots, H_{n}\right)=\{e\}$ (Proposition 2.9). Therefore, $\sigma=\sigma^{\prime}$ and so $\mathbb{E}_{n}=\mathbb{Q}_{n}$.

\section{Acknowledgements}

The work is partly supported by the Royal Society and EPSRC.

\section{References}

[1] V. V. Bavula, Every monomorphism of the Lie algebra of triangular polynomial derivations is an automorphism, C. R. Acad. Sci. Paris, Ser. I, 350 (2012) no. 11-12, 553-556. (Arxiv:math.AG:1205.0797).

[2] V. V. Bavula, Lie algebras of triangular polynomial derivations and an isomorphism criterion for their Lie factor algebras, Izvestiya: Mathematics, (2013), in print. (Arxiv:math.RA:1204.4908).

[3] V. V. Bavula, The groups of automorphisms of the Lie algebras of triangular polynomial derivations, Arxiv:math.AG/1204.4910.

[4] V. V. Bavula, The group of automorphisms of the Lie algebra of derivations of a polynomial algebra. Arxiv:math.RA:1304.6524.

[5] V. V. Bavula, The groups of automorphisms of the Witt $W_{n}$ and Virasoro Lie algebras. Arxiv:math.RA:1304.6578.

Department of Pure Mathematics

University of Sheffield

Hicks Building

Sheffield S3 7RH

UK

email: v.bavula@sheffield.ac.uk 